\title{
Excitonic Polaritons in Transverse Magnetic Fields
}

\author{
D. LOGINOV \\ V.A. Fock Institute of Physics, St. Petersburg State University \\ 198904 St. Petersburg, Russia \\ V.P. KochereshKo, A. Litvinov \\ A.F. Ioffe Physico-Technical Institute RAS \\ 194021 St. Petersburg, Russia \\ L. Besombes, H. Mariette \\ Laboratoire de Spectrométrie Physique \\ Université Joseph Fourier de Grenoble, France \\ J.J. Davies, L.C. Smith and D. Wolverson \\ Department of Physics, University of Bath, BA2 7AY Bath, UK \\ Reflectivity spectra taken from wide quantum wells based on \\ $\mathrm{CdTe} / \mathrm{CdZnTe}$ and GaAs/AlGaAs (in which the well width is much larger \\ than the exciton Bohr radius) were studied in magnetic fields in the plane \\ of the well (the Voigt geometry). The energy splitting between bright and \\ dark excitons and the redistribution of the oscillator strength from bright \\ to dark was observed and this allowed us to determine the exciton exchange \\ constant $\Delta$. We found that exciton translational mass depends on the value \\ of magnetic field. Additionally, we found that diamagnetic shift also depends \\ on exciton wave vector. Changes in the spectra in the range of the overlap \\ of heavy and light hole exciton resonances were observed when the magnetic \\ field was inverted. Theoretical modeling of the spectra allowed us to explain \\ all the observed features in the reflectivity spectra and to determine some \\ material parameters.
}

PACS numbers: 71.35.-y, 71.35.Ji, 78.20.Ls, 78.67.De

\section{Introduction}

Exciton states in bulk materials have been well studied theoretically and experimentally in different semiconductor compounds and under different external 
fields. Because only excitons with near-zero wave vector in bulk can interact with light, these studies were mainly limited to the case of excitons at rest or excitons with very small momentum. In contrast, wide quantum wells provide a unique opportunity to study excitonic states with nonzero wave vectors, i.e. excitons in motion $[1,2]$. Due to size quantization, features of exciton states with nonzero wave vector emerge in optical spectra [3]. Exciton motion in a magnetic field is particularly interesting for fundamental science because many new optical effects can be expected. The first study of exciton motion in a magnetic field was reported in the classical paper of Ref. [4], in which a notable phenomenon caused by the inversion of the magnetic field was observed, namely a difference between the spectra obtained for opposite field directions. However, there were no further detailed studies of exciton motion in magnetic fields until recently, when, in the paper [5], a new phenomenon of motional enhancement of the exciton magnetic moment was found in magnetic fields normal to the layer plane (the Faraday geometry) for excitons in wide quantum wells.

In the present paper we studied exciton states in wide quantum wells based on CdTe/CdZnTe and GaAs/AlGaAs, but with the magnetic field in the plane of the quantum well (the Voigt geometry).

\section{Results and discussion}

We used $\mathrm{CdTe} / \mathrm{Cd}_{0.96} \mathrm{Zn}_{0.04} \mathrm{Te}$ and $\mathrm{GaAs} / \mathrm{Al}_{0.3} \mathrm{Ga}_{0.7} \mathrm{As}$ single quantum well (QW) heterostructures. The structures were grown by MBE in the [100] direction. The QW width was $66 \mathrm{~nm}$ and $260 \mathrm{~nm}$ in the CdTe and GaAs structures, respectively, which is much larger than the exciton Bohr radius in these materials (6 $\mathrm{nm}$ and $13 \mathrm{~nm}$, respectively). The CdTe structure was strained due to lattice mismatch, whilst the GaAs structure was unstrained. Consequently, in the CdTe-based structure the energies of the light hole and heavy hole excitons with zero translational momentum were split apart by $20 \mathrm{meV}$, whereas in the GaAs structure they were degenerate. Reflectivity spectra were measured at $2 \mathrm{~K}$ at normal incidence in magnetic fields in the Voigt geometry for two opposite directions of the magnetic fields $\left(B^{+}\right.$and $\left.B^{-}\right)$.

Figure 1 shows reflectivity spectra taken from the $66 \mathrm{~nm} \mathrm{CdTe/CdZnTe}$ QW. Pronounced oscillations were observed in these spectra at energies above $1.590 \mathrm{eV}$. These oscillations are connected with the center of mass quantization of the optically active (bright) exciton state [2] (Fig. 1a).

In magnetic fields these oscillations become more pronounced and new oscillations emerge when the magnetic field is sufficiently high. Each of these new oscillations emerges as a splitting of each of the zero field oscillations. The amplitude of the new oscillations and their separation from the bright exciton lines increases with the growth of the magnetic field (Fig. 1b).

It is known that a transverse magnetic field mixes optically active bright exciton states (with angular momentum \pm 1 ) with optically inactive dark states 


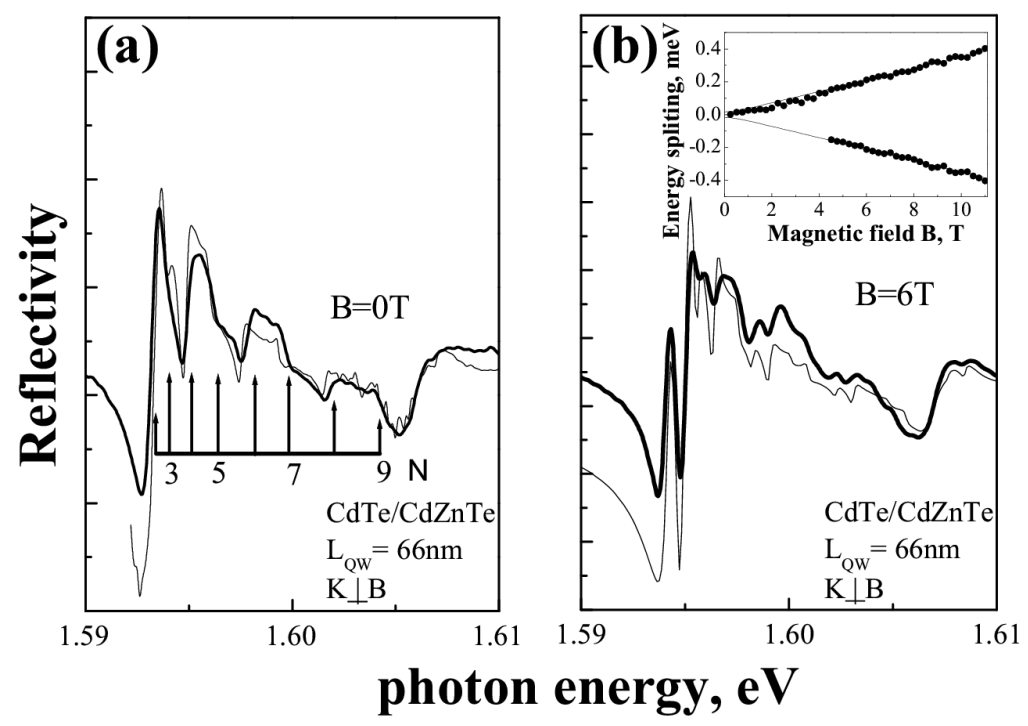

Fig. 1. Reflectivity spectra taken from $66 \mathrm{~nm} \mathrm{CdTe/CdZnTe} \mathrm{QW} \mathrm{in} \mathrm{(a)} \mathrm{zero} \mathrm{magnetic}$ field, (b) magnetic field $B=6 \mathrm{~T}$. The magnetic field is applied in the plane of the QW. Arrows indicate exciton quantized states $N$. Numbers $N$ correspond to the optically active "bright" exciton states. Solid line indicates the experimental values, dotted line - calculation. Inset shows a splitting of bright and dark exciton states.

(with angular momentum \pm 2 ). It is natural to link these additional oscillations to magnetic field mixing of dark and bright excitons. When the magnetic field is in the plane of the QW, the energy separation between dark and bright exciton states increases with magnetic field $B$ as

$$
\Delta E=\sqrt{\Delta^{2}+\left(\mu_{\mathrm{B}} g_{\mathrm{e}} B\right)^{2}},
$$

where $\Delta$ is the exciton exchange splitting, $\mu_{\mathrm{B}}$ is the Bohr magneton and $g_{\mathrm{e}}$ is the electron $g$-factor.

In the reflectivity spectra we observed that each of the quantized exciton states splits according to Eq. (1) (inset to Fig. 1b), so that the exchange constant $\Delta$ can be measured. The constant $\Delta$ is found to be the same for all quantized states to within experimental accuracy.

We performed calculations of these spectra as in paper [3]. The results of the calculations are shown by dotted lines in Figs. 1a and b. For CdTe we found $\Delta=$ $0.03 \mathrm{meV}, g_{\mathrm{e}}=1.5$, and, for GaAs, $\Delta=0.02 \mathrm{meV}, g_{\mathrm{e}}=-0.45$. Such parameters were discussed theoretically in [6] and measured in several publications. (There is a distribution of the published values. It was found for CdTe $\Delta=0.6 \mathrm{meV}$ in [7] and $\Delta=0.07 \mathrm{meV}$ in [8], for GaAs $\Delta=0.37 \mathrm{meV}$ in [9] and $\Delta=0.05 \mathrm{meV}$ in [10].) The values of $g$-factors in CdTe and GaAs coincide with our data and are presented in [11] and [12]. We believed that our data are very close to be true. 
The dependence of the exciton translational mass on magnetic field has been found. This dependence displays in the experiment, as the interference structure of exciton quantization in reflectivity spectra becomes condensed in magnetic fields. Fitting the calculated spectra to the experimental data allows us to plot the dependence of exciton mass on magnetic fields (Fig. 2a). One can see that this dependence is linear in magnetic field $B, M=M(0)(1+\alpha B)$. Here $\alpha$ is a certain constant and $M(0)=0.8 m_{0}$ ( $m_{0}$ is the free electron mass) is the exciton translation mass in zero field. We find $\alpha=0.0267 \mathrm{~T}^{-1}$.
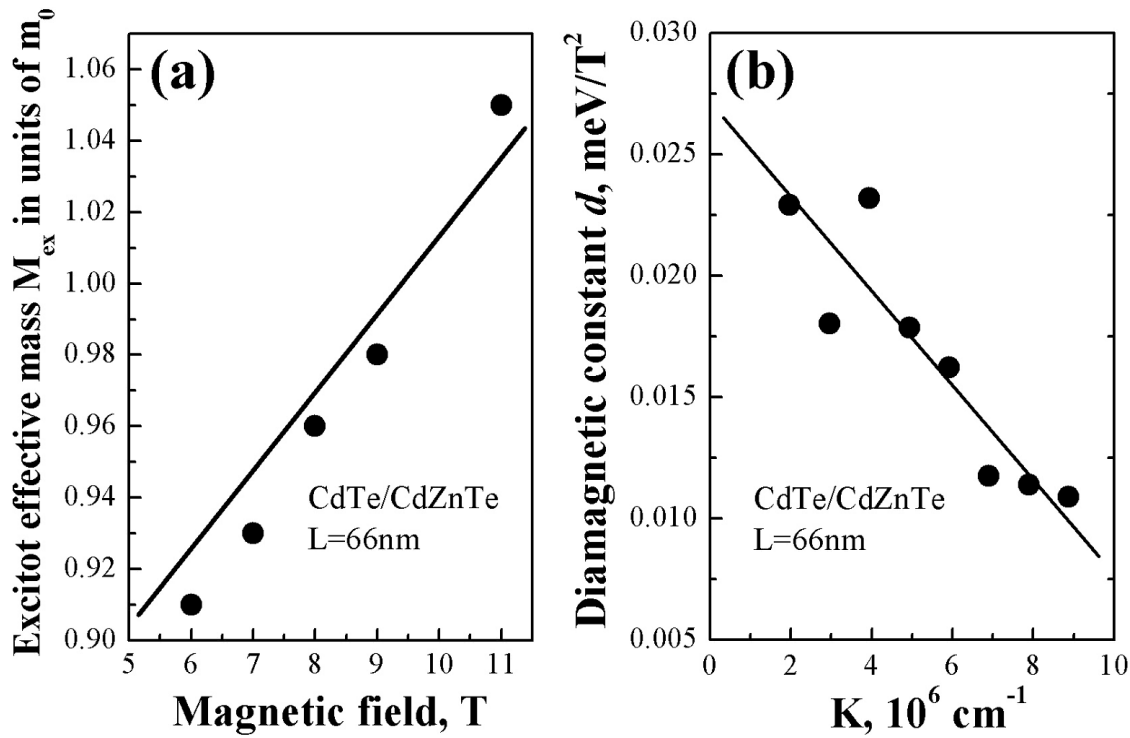

Fig. 2. (a) Dependence of exciton translation mass on magnetic field determined from exciton-polariton dispersion curves in magnetic fields. (b) Dependence of diamagnetic constant $d$ on the quantization number of the exciton level.

In addition to the exciton translational mass dependence we found the dependence of exciton diamagnetic constant $d$ (the diamagnetic shift is $\Delta E=d B^{2}$ ) on the exciton wave vector, i.e. the value of the diamagnetic shift is different for different quantized states. This dependence is shown in Fig. $2 \mathrm{~b}$ and is seen to be linear: $d=d_{0}-\beta K$. Here $K$ is the exciton translational wave vector, $d_{0}$ is the diamagnetic constant for excitons with $K=0$ and $\beta$ is a certain constant. We find $d_{0}=0.0275 \mathrm{meV} \mathrm{T}^{-2}$ and $\beta=1.95 \times 10^{-9} \mathrm{~cm} \mathrm{meV} \mathrm{T}^{-2}$.

According to Fig. 2a in a small energy diapason we can express the obtained dependence of the exciton energy on the effective mass as $\frac{\hbar^{2} K^{2}}{2 M}(1-\gamma B)$. Consequently, the exciton energy contains a contribution proportional to $K^{2} B$ and according to Fig. $2 \mathrm{~b}$ it contains a contribution proportional to $K B^{2}$. Both these dependences arise from the terms in the electron and hole Hamiltonians which are cubic in wave vector, namely the terms $\propto \sum_{i} \sigma_{i} k_{i}\left(k_{i+1}^{2}-k_{i+2}^{2}\right)$ (electrons) and 
$\propto \sum_{m} J_{m} k_{m}\left(k_{m+1}^{2}-k_{m+2}^{2}\right)$ (holes). In the presence of magnetic fields we have to replace $k_{i}$ in these formulae by $k_{i}+\frac{e}{c} A_{i}$, where $A_{i}$ are components of the vector potential. This will give additional contributions to the diamagnetic shift and exciton effective mass. Consequently both effects presented in Fig. 2a, b are of the same origin, namely the terms which are cubic in wave vector.

In the Voigt geometry we observed an effect of magnetic field inversion [1, 2], consisting of a difference between the reflectivity spectra for magnetic fields $+B$ and $-B$ in opposite directions (see Fig. 3a, b). This effect was strongest in the region in which heavy and light exciton transitions coexist and is apparently caused by mixing of light and heavy excitons by the magnetic field.

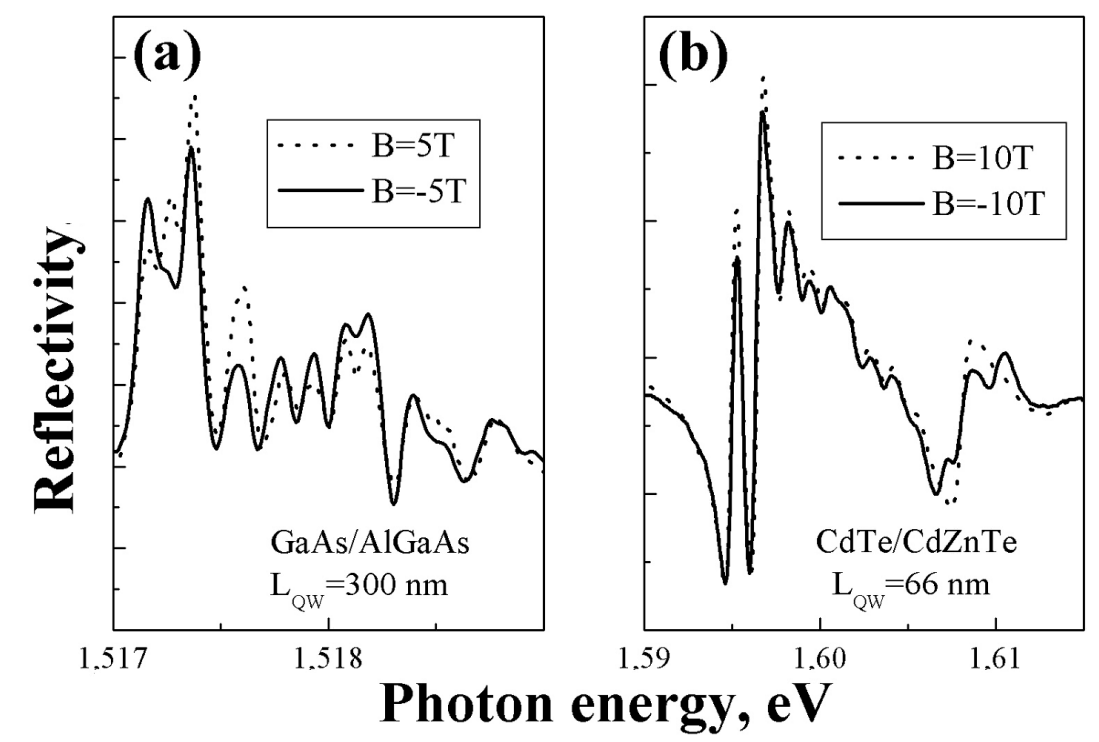

Fig. 3. "Effect of magnetic field inversion" in the quantum well structures with wide QW based on GaAs (a) and CdTe (b). Solid line: $-B$, dotted line: $+B$ (two opposite directions of magnetic field).

This effect due to magnetic field inversion was studied for the first time in $[1,2]$ and also in [13]. It was described by terms in the exciton Hamiltonian such as $\propto \boldsymbol{K} \times \boldsymbol{B}$, which mix optically active $1 S$ and optically inactive $2 P$ exciton states [14]. The present experiments indicate that the effect of inversion can also emerge from mixing between heavy and light hole exciton states. The behavior appears to be due to $(\boldsymbol{J} \cdot \boldsymbol{K})^{2}$ terms in the Luttinger Hamiltonian, which split heavy and light holes. This question is now under a further experimental and theoretical study. 


\section{Conclusion}

In wide QWs we observed a splitting of bright and dark excitons and redistribution of the oscillator strength from bright to dark states. This allowed us to determine the exciton exchange constant $\Delta$ in $\mathrm{CdTe}$ and in GaAs. For the first time we found that the exciton translational mass depends on the value of the magnetic field. Additionally, we found that the diamagnetic shift depends on exciton wave vector.

Changes in the spectra caused by inversion of the magnetic field were observed for CdTe and GaAs in the Voigt geometry. Theoretical modeling of the spectra allowed us to explain all the observed features in the reflectivity spectra and to determine some material parameters.

\section{Acknowledgments}

The work was supported in part by grants from RFBR, Presidium RAS, the Royal Society, EPSRC (UK), and CNRS.

\section{References}

[1] V.A. Kiselev, B.S. Razbirin, I.N. Uraltsev, Phys. Status Solidi B 72, 161 (1975).

[2] H. Tuffigo, R.T. Cox, N. Magnea, Y. Merle d'Aubigné, A. Million, Phys. Rev. B 37, 4310 (1988).

[3] D.K. Loginov, E.V. Ubyivovk, Yu.P. Efimov, V.V. Petrov, S.A. Eliseev, Yu.K. Dolgikh, I.V. Ignatiev, V.P. Kochereshko, A.V. Selkin, Phys. Solid State 48, 2100 (2006).

[4] D.G. Thomas, J.J. Hopfield, Phys. Rev. 124, 657 (1961) and E.F. Gross, B.P. Zakharchenia, O.V. Konstantinov, Sov. Phys.-Solid State 3, 305 (1961).

[5] J.J. Davies, D. Wolverson, V.P. Kochereshko, A.V. Platonov, R.T. Cox, J. Cibert, H. Mariette, C. Bodin, C. Gourgon, E.V. Ubyivovk, Yu.P. Efimov, S.A. Eliseev, Phys. Rev. Lett. 97, 187403 (2006).

[6] M. Suffczynski, L. Swierkowski, W. Wardzynski, J. Phys. C, Solid State Phys. 8, L52 (1975).

[7] D.D. Sell, Surf. Sci. 35, 863 (1973).

[8] W. Wardzyński, M. Suffczyński, Solid State Commun. 10, 417 (1972).

[9] M.A. Gilleo, P.T. Bailey, D.E. Hill, Phys. Rev. 174, 898 (1968).

[10] K. Cho, W. Dreybrodt, P. Hiesinger, S. Suga, F. Willmann, in: Proc. 12th Int. Conf. on the Physics of Semiconductors, Stuttgart 1974, Ed. M.H. Pilkuhn, B.G. Toubner, Stuttgart 1974, p. 945.

[11] A.A. Sirenko, T. Ruf, M. Cardona, D.R. Yakovlev, W. Ossau, A. Waag, G. Landwehr, Phys. Rev. B 56, 2114 (1997).

[12] C. Hermann, C. Weisbuch, Phys. Rev. B 15, 823 (1977).

[13] V.P. Kochereshko, G.V. Mikhailov, I.N. Uraltsev, Sov. Phys.-Solid State 25, 769 (1983).

[14] R.S. Knox, Theory of Excitons, Academic Press, New York 1963. 